\title{
The Trend of Psychiatric Visits and Psychiatric Medication Prescription Among People Tested for SARS-CoV-2 During the Initial Phase of COVID-19 Pandemic in South Korea
}

\author{
Jungeun Kim¹, Soo Jung Rim¹, Minkyung Jo ${ }^{1}$, Min Geu Lee ${ }^{1}$, and Subin Park ${ }^{2}$ \\ ${ }^{1}$ Department of Research Planning, Mental Health Research Institute, National Center for Mental Health, Seoul, Republic of Korea \\ ${ }^{2}$ Mental Health Research Institute, National Center for Mental Health, Seoul, Republic of Korea
}

Objective The objective of this study was to monitor the trend of psychiatric visits and medication prescriptions among people tested for SARS-CoV-2 during the initial phase of coronavirus disease 2019 (COVID-19) pandemic in South Korea.

Methods We conducted a population-based cohort study using data from the National Health Insurance Service (NHIS) linked to the Korea Disease Control and Prevention Agency (KDCA) data. The overall trend of psychiatric visits during COVID-19 for each month was compared to the month prior to COVID-19. The number of psychiatric medication prescription records was monitored from January 2019 to May 2020.

Results A total of 212,678 individuals were tested for SARS-CoV-2 between January 2020 and May 2020. Among these individuals, $72.1 \%(n=153,309)$ did not have pre-existing mental illness, and $27.9 \%(n=59,369)$ had pre-existing mental illness. We found that most psychiatric visits were made in March, and patients without pre-existing mental illness significantly increased in psychiatric outpatients during the COVID-19 pandemic ( $p$ trend $<0.001$ ). In addition, psychiatric medication prescriptions were the most prescribed between March and April 2020.

Conclusion We identified a rising trend in psychiatric visits and medication prescriptions among people who were tested for SARSCoV-2 during the initial phase of COVID-19 pandemic in South Korea.

Psychiatry Investig 2022;19(1):61-71

Keywords COVID-19; Mental health; Outpatient; Inpatient; South Korea.

\section{INTRODUCTION}

Coronavirus disease 2019 (COVID-19), caused by the severe acute respiratory syndrome coronavirus 2 (SARS-CoV-2), first emerged in December 2019 and rapidly developed into a global pandemic that continues to progress in terms of an increase in the number of confirmed cases and mortality worldwide. Consequently, the World Health Organization (WHO) declared a global pandemic in March 2020. ${ }^{1}$ In South Korea ("Korea" from here on), the first COVID-19 case was confirmed on January 20, 2020 and within two months, an exponential increase was observed in terms of confirmed COVID-19 cases. As of March 31, 2020, a total of 9,786 confirmed cases of

Received: July 27, 2021 Revised: October 28, 2021

Accepted: November 27, 2021

$\square$ Correspondence: Subin Park, MD, PhD

Mental Health Research Institute, National Center for Mental Health, 127 Yongmasan-ro, Gwangjin-gu, Seoul 04933, Republic of Korea

Tel: +82-2-2204-0103, Fax: +82-2-2204-0362, E-mail: subin-21@hanmail.net

(c) This is an Open Access article distributed under the terms of the Creative Commons Attribution Non-Commercial License (https://creativecommons.org/licenses/by$\mathrm{nc} / 4.0$ ) which permits unrestricted non-commercial use, distribution, and reproduction in any medium, provided the original work is properly cited.
COVID-19 had been reported in Korea. ${ }^{2}$

To counteract this crisis, the Korean government has set up screening stations in various areas to enhance access to SARS-CoV-2 tests and to identify confirmed cases through quick and extensive tests. ${ }^{3}$ Korea also controlled the spread of COVID-19 in various ways (e.g., contact tracing, quarantine, isolation, social distancing, and school closures). ${ }^{4}$ Through the aforementioned efforts, the Korean government was able to control COVID-19 quickly and effectively, but it greatly affected the mental health of the general population. ${ }^{5}$

Several factors of COVID-19, such as uncertainty of prognosis, limited professionals and equipment, restrictions on traveling, and conflicting messages from authorities, are worrisome with respect to the mental health of the population. ${ }^{6}$ In particular, the prolonged restrictions on social interactions that the population is going through are associated with worsened psychiatric symptoms such as panic, stress, anxiety, depression, and insomnia. ${ }^{7.8}$

These findings are in line with those of previous studies ${ }^{9-12}$ showing worsened mental health during other epidemics such 
as SARS or Ebola virus outbreaks. In particular, psychiatric symptoms, such as posttraumatic stress symptoms, anxiety, depression, suicide, and substance use, were elevated during SARS and Ebola virus outbreaks and lasted for more than 30 months. ${ }^{13,14}$

According to a systematic review regarding mental health and COVID- $19,{ }^{15}$ the general population, patients with COVID-19, health care workers, and psychiatric patients (diagnosed before COVID-19) showed worsened mental health. Additionally, in one study, ${ }^{16}$ about $25 \%$ and $22 \%$ of new and existing psychiatric patients, respectively, faced a barrier in receiving a timely diagnosis or routine care. Moreover, in the same study, about $20 \%$ of those with preexisting psychiatric disorder before COVID-19 reported that their mental health condition worsened. Therefore, it is important to monitor the psychiatric visits of the general population and patients with pre-existing psychiatric disorders during the COVID-19 pandemic in Korea. In particular, people who tested for SARSCoV-2 are at a high risk of mental problems, such as the fear of infection and concerns about health, stigma, and economic loss. ${ }^{17}$ Several studies have shown that people who tested positive for SARS-CoV-2 experienced more mental illnessrelated problems such as posttraumatic stress symptoms ${ }^{7}$ and depression. ${ }^{18}$ Therefore, it is necessary to monitor trends in the incidence and deterioration of mental health problems.

Previous studies on mental health problems during COVID-19 have utilized self-administered surveys ${ }^{19-23}$ or have relied on hospital data, ${ }^{24}$ rather than nationally representative data. Therefore, studies on the actual diagnosis of mental illness using nationally representative data during COVID-19 are limited. The objective of this study was to monitor the monthly trend of psychiatric visits along with psychiatric medication prescriptions among people tested for SARS-CoV-2 during the initial phase of COVID-19 pandemic in Korea. In particular, the current study tried to identify the onset of new psychiatric disorders and the worsening of existing psychiatric disorders by separately investigating those who did not have psychiatric disorders and those who had them before the onset of the COVID-19 pandemic.

\section{METHODS}

\section{Data source and study population}

Korea has a national health insurance system that includes $98 \%$ of the nation's population; the patient database is recorded based on ICD-10 codes. ${ }^{25}$ We conducted a population-based cohort study using National Health Insurance claims data from the National Health Insurance Service (NHIS) linked to the Korea Disease Control and Prevention Agency (KDCA) data. The KDCA provides data regarding people who were tested for SARS-CoV-2 at public health centers and medical centers from January 1, 2020, to May 31, 2020. The NHIS data contains outpatient and inpatient demographic information and healthcare records, such as diagnoses, prescription medications, procedures, and health care visits. ${ }^{25}$

We included 212,678 individuals aged 20 years or older who were tested for SARS-CoV-2 between January 1 and May 31, 2020. SARS-CoV-2 testing was confirmed by laboratory testing with real-time reverse transcription polymerase chain reaction (RT-PCR) assays of nasal and pharyngeal swabs, as recommended by the WHO. ${ }^{26}$ This study was approved by the Institutional Review Board of the National Center for Mental Health (116271-2020-46).

\section{Measures}

We defined patients with a pre-existing mental illness as those who had a diagnostic code for mental and behavioral disorders (ICD-10 codes: F00-F99, G47) at least once within the 1-year period prior to January 2020.

We classified medication into four main categories: antianxiety, antidepressants, sedative-hypnotics, and antipsychotics. Sedative-hypnotic medications included Z-drugs, benzodiazepines, and other medications prescribed for off-label uses (antidepressants: imipramine, amitriptyline, low-dose formulation mirtazapine, trazodone, and nortriptyline; and low-dose antipsychotics: levomepromazine, chlorpromazine, quetiapine). ${ }^{27}$ The number of psychiatric medication prescription records was monitored for each of the four medication categories from January 2019 to May 2020.

Demographic variables included gender, age (20-29, 30$39,40-49,50-59,60-69,70-79, \geq 80$ ). Region of residence (Seoul, Daegu, Gyeonggi-do, Gyeongsangbuk-do, other), income level (low, medium, high, unknown), and insurance type (self-employed, employee, medical aid).

\section{Statistical analysis}

The baseline characteristics of the entire individuals included in this study were demonstrated as percentages for categorical variables. A chi-square test for categorical variables was used to compare the differences between the groups with and without pre-existing mental illness, and also the differences between the negative and positive results of SARS-CoV-2 test. The overall trend of psychiatric visits during COVID-19 for each month was compared to the same month prior to COVID-19 (e.g., the trend of May 2020 was compared with the trend of May 2019). ${ }^{28}$ The number of visits between patients with and without pre-existing mental illness was confirmed to be significant using $\mathrm{p}$ for trend among, and trends were analyzed in detail according to the results of the SARS-CoV-2 test. All statistical analyses were conducted using SAS (version 9.4; 
SAS Institute Inc., Cary, NC, USA), and p-values less than or equal to 0.05 were considered statistically significant.

\section{RESULTS}

A total of 212,678 individuals were tested for SARS-CoV-2 between January 2020 and May 2020. Among these individuals, $72.1 \%(n=153,309)$ did not have pre-existing mental illness, and $27.9 \%(\mathrm{n}=59,369)$ had pre-existing mental illness. The total number of women was 112,640 (53\%). Among pa- tients without pre-existing mental illness, the age group of 20-29 years showed the highest proportion (22.8\%), and among patients with pre-existing mental illness, those aged 80 years or older showed the highest proportion (23.5\%). In total, 25\% of those who were tested for SARS-CoV-2 had a psychiatric visit record between January and May 2020. Among those without pre-existing mental illness, 14,864 (9.7\%) and 39,026 (65.7\%) patients with pre-existing mental illness had a psychiatric visit (Table 1 ).

Of the total 212,678 patients who were tested for the SARS-

Table 1. Baseline characteristics of individuals tested for SARS-CoV-2

\begin{tabular}{|c|c|c|c|c|}
\hline Variables & $\begin{array}{c}\text { Total } \\
(\mathrm{N}=212,678)\end{array}$ & $\begin{array}{c}\text { Without pre-existing } \\
\text { mental illness }(\mathrm{N}=153,309)\end{array}$ & $\begin{array}{c}\text { With pre-existing } \\
\text { mental illness }(\mathrm{N}=59,369)\end{array}$ & p-value \\
\hline Gender & & & & $<0.001$ \\
\hline Male & $100,038(47.0)$ & $74,193(48.4)$ & $25,845(43.5)$ & \\
\hline Female & $112,640(53.0)$ & $79,116(51.6)$ & $33,524(56.5)$ & \\
\hline Age group & & & & $<0.001$ \\
\hline $20-29$ & $39,563(18.6)$ & $34,960(22.8)$ & $4,603(7.8)$ & \\
\hline $30-39$ & $38,809(18.2)$ & $33,394(21.8)$ & $5,415(9.1)$ & \\
\hline $40-49$ & $32,397(15.2)$ & $26,509(17.3)$ & $5,888(9.9)$ & \\
\hline $50-59$ & $30,660(14.4)$ & $22,664(14.8)$ & $7,996(13.5)$ & \\
\hline $60-69$ & $27,098(12.7)$ & $17,244(11.2)$ & $9,854(16.6)$ & \\
\hline $70-79$ & $22,704(10.7)$ & $11,024(7.2)$ & $11,680(19.7)$ & \\
\hline $80+$ & $21,447(10.1)$ & $7,514(4.9)$ & $13,933(23.5)$ & \\
\hline Region of residence & & & & $<0.001$ \\
\hline Seoul & $46,419(21.8)$ & $33,904(22.1)$ & $12,515(21.1)$ & \\
\hline Daegu & $25,787(12.1)$ & $19,002(12.4)$ & $6,785(11.4)$ & \\
\hline Gyeonggi-do & $51,314(24.1)$ & $38,122(24.9)$ & $13,192(22.2)$ & \\
\hline Gyeongsangbuk-do & $13,276(6.2)$ & $8,750(5.7)$ & $4,526(7.6)$ & \\
\hline Other & $75,882(35.7)$ & $53,531(34.9)$ & $22,351(37.6)$ & \\
\hline Income level & & & & $<0.001$ \\
\hline Low & $55,455(26.1)$ & $35,621(23.2)$ & $19,834(33.4)$ & \\
\hline Medium & $62,938(29.6)$ & $48,751(31.8)$ & $14,187(23.9)$ & \\
\hline High & $90,717(42.7)$ & $66,223(43.2)$ & $24,494(41.3)$ & \\
\hline Unknown & $3,568(1.7)$ & $2,714(1.8)$ & $854(1.4)$ & \\
\hline Insurance type & & & & $<0.001$ \\
\hline Self-employed & $48,602(22.9)$ & $32,759(21.4)$ & $15,843(26.7)$ & \\
\hline Employee & $152,225(71.6)$ & $116,243(75.8)$ & $35,982(60.6)$ & \\
\hline Medical aid & $11,851(5.6)$ & $4,307(2.8)$ & $7,544(12.7)$ & \\
\hline Test results of SARS-CoV-2 & & & & $<0.001$ \\
\hline Positive & $7,713(3.6)$ & $5,962(3.9)$ & $1,751(2.9)$ & \\
\hline Negative & $204,965(96.4)$ & $147,347(96.1)$ & $57,618(97.1)$ & \\
\hline Psychiatric visit* & & & & $<0.001$ \\
\hline Yes & $53,890(25.3)$ & $14,864(9.7)$ & $39,026(65.7)$ & \\
\hline No & $158,788(74.7)$ & $138,445(90.3)$ & $20,343(34.3)$ & \\
\hline
\end{tabular}

*psychiatric visit: the number of psychiatric visit (inpatient, outpatient) between January and May 2020. SARS-CoV-2, severe acute respiratory syndrome coronavirus 2 
CoV-2, 7,713 (3.6\%) tested positive. The highest proportion of SARS-CoV-2 positive patients was in Daegu region (65.3\%). The proportion of people who had pre-existing mental illnesses was slightly higher in people who tested negative compared to those who tested positive (28.1\% vs. $22.7 \%$ ) (Table 2 ).

Figure 1 shows the number of SARS-CoV-2 tests per month and the cumulative tests performed from January to May 2020. As shown in Figure 1, the number of SARS-CoV-2 tests steeply increased in March (approximately 2.5 times compared to February) and started to decline from April.

Figure 2 shows the trend in the number of psychiatric visits from January 2019 to May 2020. It illustrates the number of total (both inpatient and outpatient), inpatient, and outpatient psychiatric visits. The number of outpatient visits was the highest at 41,664 in March 2020, which increased by $19.8 \%$, com- pared to the same month of the previous year (i.e., March 2019). In addition, the number of inpatient visits was the highest at 11,406 in April, an increase of more than 200\% compared to

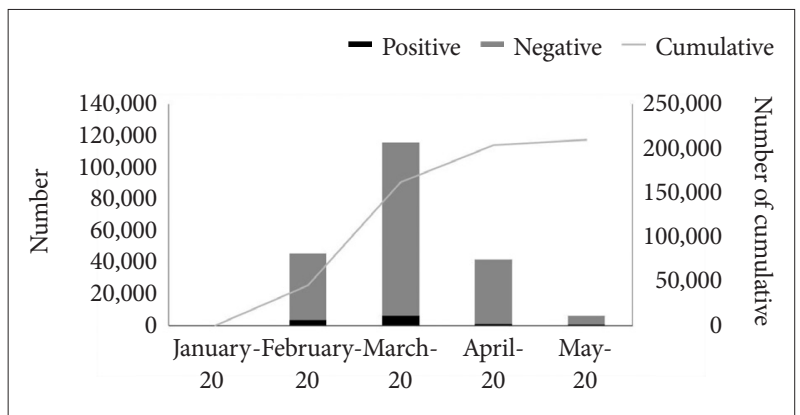

Figure 1. Number of SARS-CoV-2 tests per month and cumulative tests performed from January 2020 to May 2020. SARS-CoV-2, severe acute respiratory syndrome coronavirus 2 .

Table 2. Baseline characteristics of individuals according to SARS-CoV-2 test result

\begin{tabular}{|c|c|c|c|c|}
\hline Variables & Total $(\mathrm{N}=212,678)$ & Negative $(N=204,965)$ & Positive $(\mathrm{N}=7,713)$ & $\mathrm{p}$-value \\
\hline Gender & & & & $<0.001$ \\
\hline Male & $100,038(47.0)$ & $96,990(47.3)$ & $3,048(39.5)$ & \\
\hline Female & $112,640(53.0)$ & $107,975(52.7)$ & $4,665(60.5)$ & \\
\hline Age group & & & & $<0.001$ \\
\hline $20-29$ & $39,563(18.6)$ & $37,506(18.3)$ & $2,057(26.7)$ & \\
\hline $30-39$ & $38,809(18.2)$ & $37,977(18.5)$ & $832(10.8)$ & \\
\hline $40-49$ & $32,397(15.2)$ & $31,361(15.3)$ & $1,036(13.4)$ & \\
\hline $50-59$ & $30,660(14.4)$ & $29,093(14.2)$ & $1,567(20.3)$ & \\
\hline $60-69$ & $27,098(12.7)$ & $25,899(12.6)$ & $1,199(15.5)$ & \\
\hline $70-79$ & $22,704(10.7)$ & $22,087(10.8)$ & $617(8.0)$ & \\
\hline $80+$ & $21,447(10.1)$ & $21,042(10.3)$ & $405(5.3)$ & \\
\hline Region of residence & & & & $<0.001$ \\
\hline Seoul & $46,419(21.8)$ & $45,909(22.4)$ & $510(6.6)$ & \\
\hline Daegu & $25,787(12.1)$ & $20,751(10.1)$ & $5,036(65.3)$ & \\
\hline Gyeonggi-do & $51,314(24.1)$ & $50,883(24.8)$ & $431(5.6)$ & \\
\hline Gyeongsangbuk-do & $13,276(6.2)$ & $12,343(6.0)$ & $933(12.1)$ & \\
\hline Other & $75,882(35.7)$ & $75,079(36.6)$ & $803(10.4)$ & \\
\hline Income level & & & & $<0.001$ \\
\hline Low & $55,455(26.1)$ & $52,692(25.7)$ & $2,763(35.8)$ & \\
\hline Medium & $62,938(29.6)$ & $60,877(29.7)$ & $2,061(26.7)$ & \\
\hline High & $90,717(42.7)$ & $87,945(42.9)$ & $2,772(35.9)$ & \\
\hline Unknown & $3,568(1.7)$ & $3,451(1.7)$ & $117(1.5)$ & \\
\hline Insurance type & & & & $<0.001$ \\
\hline Self-employed & $48,602(22.9)$ & $46,554(22.7)$ & $2,048(26.6)$ & \\
\hline Employee & $152,225(71.6)$ & $147,210(71.8)$ & $5,015(65.0)$ & \\
\hline Medical aid & $11,851(5.6)$ & $11,201(5.5)$ & $650(8.4)$ & \\
\hline Pre-existing mental illness & & & & $<0.001$ \\
\hline Yes & $59,369(27.9)$ & $57,618(28.1)$ & $1,751(22.7)$ & \\
\hline No & $153,309(72.1)$ & $147,347(71.9)$ & $5,904(77.3)$ & \\
\hline
\end{tabular}

SARS-CoV-2, severe acute respiratory syndrome coronavirus 2

64 Psychiatry Investig 2022;19(1):61-71 


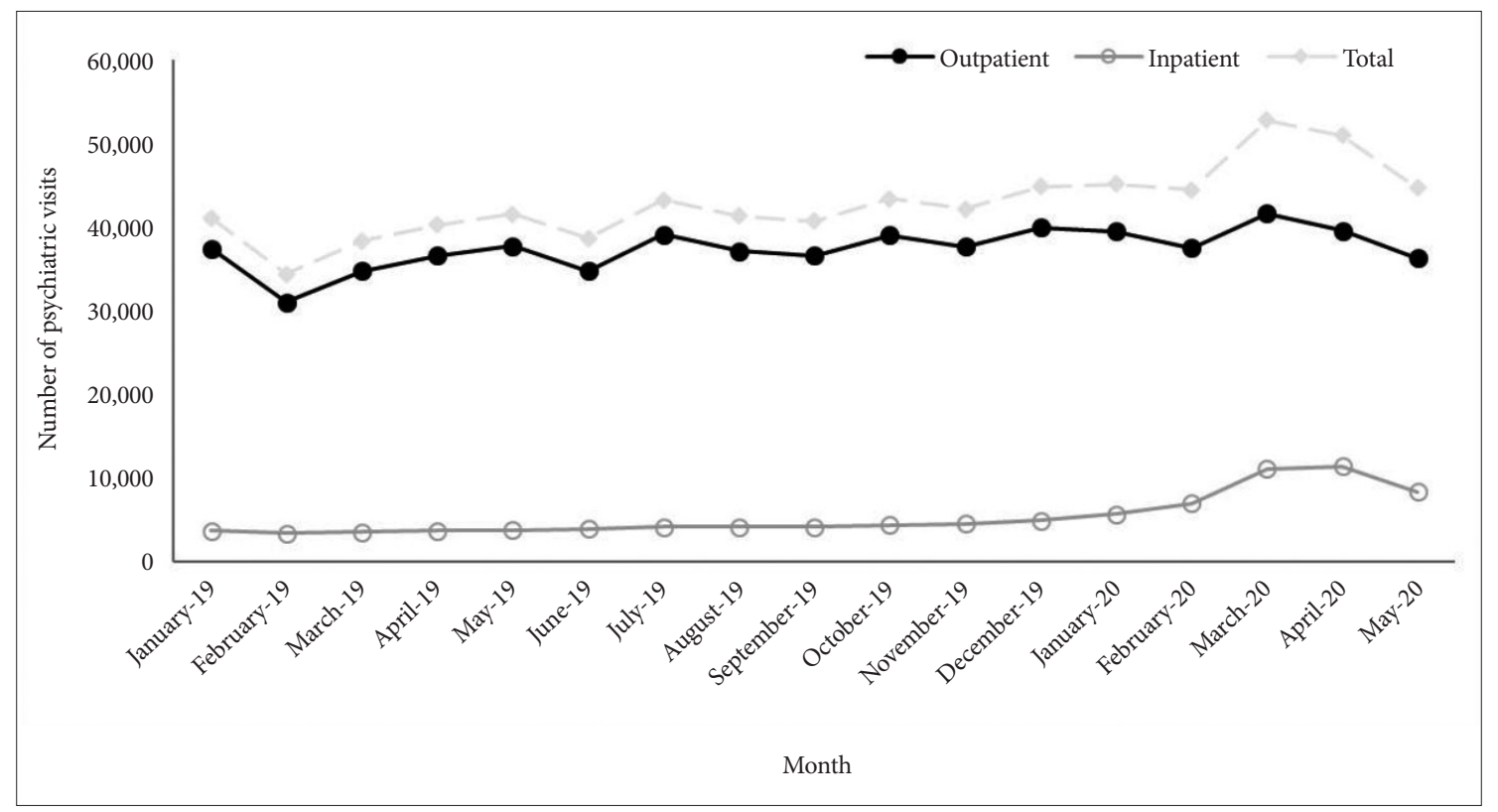

Figure 2. Number of psychiatric outpatient and inpatient visits per month from January 2019 to May 2020 among people tested for SARSCoV-2. SARS-CoV-2, severe acute respiratory syndrome coronavirus 2.

the same month of the previous year (Figure 2).

The number of outpatient visits without pre-existing mental illnesses increased significantly by April and slightly decreased in May, and the number of inpatient visits increased more than five times in April compared to January 2020 (p trend $<0.001$ ) (Figure $3 \mathrm{~A}$ ). Among the "negative for SARSCoV-2" group, the number of outpatient visits with pre-existing mental illnesses decreased slightly between January and May 2020, and the number of inpatient visits increased by April and decreased slightly in May ( $\mathrm{p}$ trend $<0.001$ ). Trends of outpatient and inpatient visits among people who tested negative for SARS-CoV-2 were nearly consistent with those in all participants (Figure 3B). Among the "positive for SARS-CoV-2" group, but did not have any pre-existing mental illnesses, the number of outpatient visits increased until April, then decreased in May ( $\mathrm{p}$ trend $<0.001$ ); however, the number of inpatient visits increased significantly until May $(\mathrm{p}$ trend $<0.001)$. In addition, among people who tested positive for SARS-CoV-2, the number of outpatient visits of those with pre-existing mental illness decreased significantly ( $p$ trend $<0.001$ ), while the number of inpatient visits increased significantly between January and May 2020 ( $p$ trend $<0.001$ ) (Figure 3C).

Furthermore, we identified trends in psychiatric medication prescriptions from January 2019 to May 2020. During the COVID-19 pandemic, prescriptions for antipsychotic, sedative-hypnosis and antianxiety medications were the highest in March 2020, which shows an increased rate of prescription of $61.0 \%, 43.4 \%$ and $38.9 \%$, respectively, compared to the same months of the previous year. Antidepressant medication prescription rate was highest in April, which is a 21.3\% increase from the same month of the previous year (Figure 4A). Among those who tested negative for SARS-CoV-2, prescriptions for psychiatric medications were peaked in March and then declined (Figure 4B). However, among people who tested positive for SARS-CoV-2, prescriptions for psychiatric medications excluding antidepressants tended to increase steadily until May (Figure 4C).

\section{DISCUSSION}

We analyzed the monthly trend of psychiatric visits along with psychiatric medication prescriptions before and during the initial phase of COVID-19 pandemic in Korea among people tested for SARS-CoV-2. In particular, we showed psychiatric visits separately for patients with and without preexisting mental illness, and for those who tested negative and positive for SARS-CoV-2 test.

We found that most psychiatric visits were made in March, when COVID-19 was declared a pandemic by the WHO. In addition, since the first case of COVID-19 in Korea on January 20,2020, the number of confirmed COVID-19 cases has exponentially increased due to the massive infection of a religious group called Shincheonji and the outbreak of COVID-19 patients in a psychiatric hospital (i.e., Cheongdo Daenam Hospital). ${ }^{29}$ Soon, Korea became the second most infected country in the world, following China at that time. In addition, community infections continued to occur in crowded facili- 


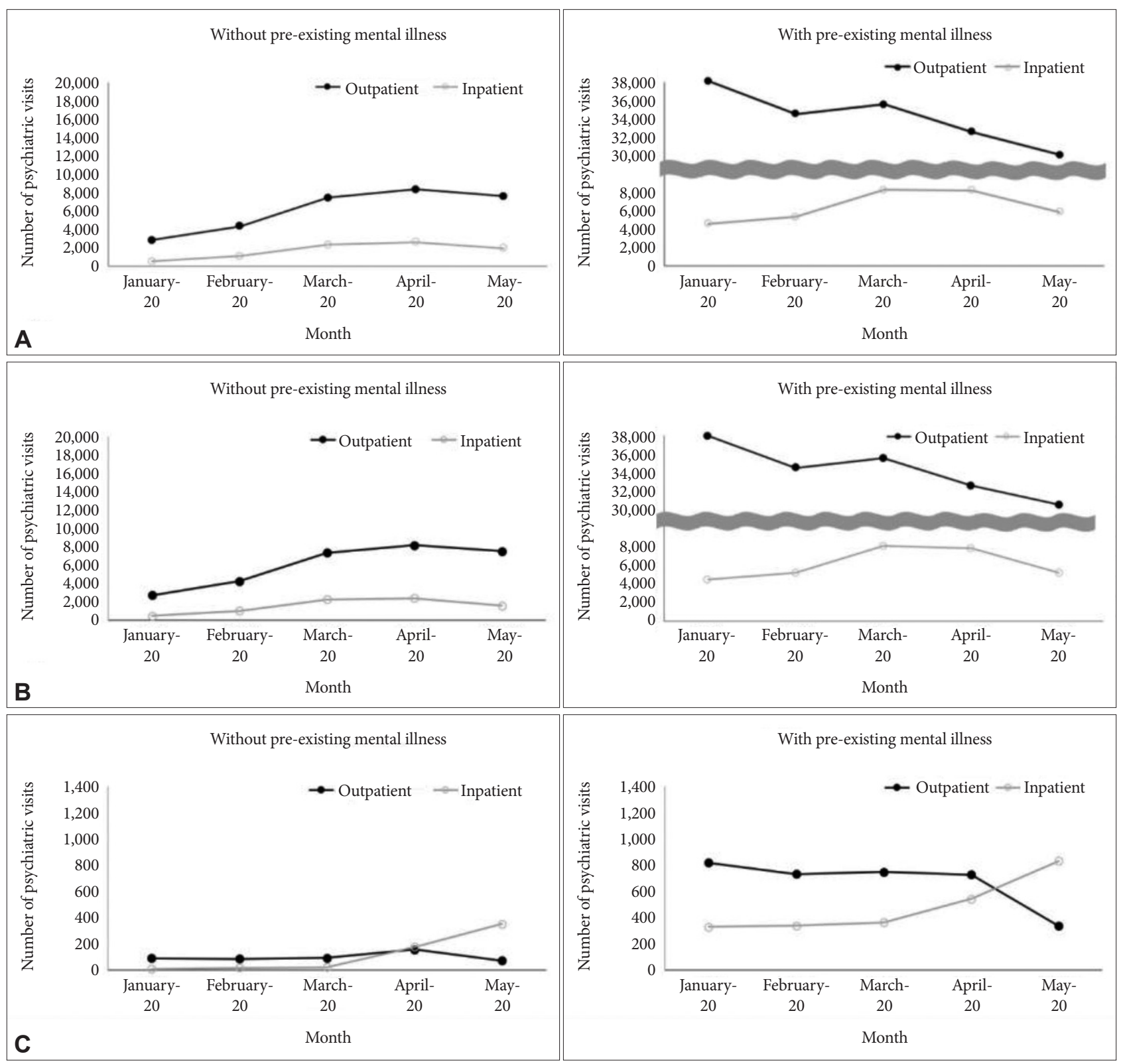

Figure 3. Number of outpatient and inpatient visits, based on the presence of pre-existing mental illness according to SARS-CoV-2 test result. A: Patients who tested for the SARS-CoV-2 test. B: Negative patients who tested for the SARS-CoV-2 test. C: Positive patients who tested for the SARS-CoV-2 test. SARS-CoV-2, severe acute respiratory syndrome coronavirus 2.

ties such as long-term care facilities, call centers, and spas. ${ }^{30-32}$ Accordingly, the Korean government had stepped up public health measures, including social distancing, quarantine, and mass diagnostic tests, to curb the virus and minimize the spread of the region in advance. ${ }^{33}$ Due to these efforts, the rapid increase in new cases has turned downward. In line with the positive COVID-19 case pattern, the number of psychiatric visits was the highest in March, and it showed a somewhat decreasing rate in April and May.

Several recent studies on mental health during the COVID-19 pandemic have shown similar results. In one study, ${ }^{5} 45 \%$ of the respondents reported clinical levels of depression, anxiety, or stress, with $12.8 \%$ at risk of mental illness during the COVID-19 pandemic. This is much higher than the baseline rate reported in previous studies conducted in Korea prior to the pandemic. Furthermore, a US study ${ }^{34}$ suggested that the prevalence of depression was more than three times higher during COVID-19 than before the onset of the pandemic.

We further analyzed the psychiatric visit trends based on the mental illness status before the COVID-19 pandemic. According to our results, the psychiatric outpatient visits of patients without pre-existing mental illness significantly increased 
J Kim et al.
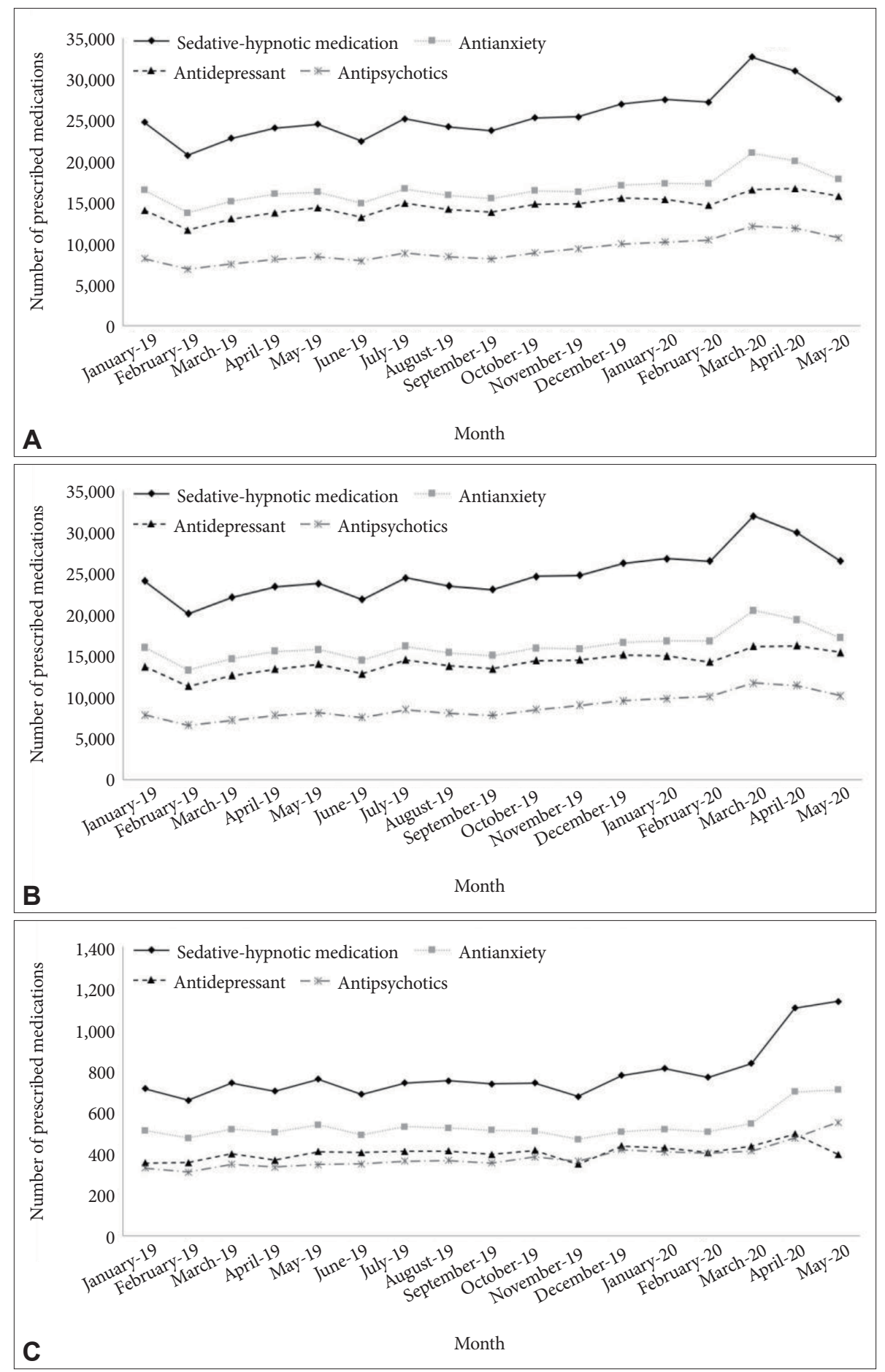

Figure 4. Number of prescribed medications per month from January 2019 to May 2020 according to SARS-CoV-2 test result. A: Patients who tested for the SARS-CoV-2 test. B: Negative patients who tested for the SARS-CoV-2 test. C: Positive patients who tested for the SARSCoV-2 test. SARS-CoV-2, severe acute respiratory syndrome coronavirus 2.

during the COVID-19 pandemic. The rate of mental health service utilization in Korea tends to be lower than that in other countries. ${ }^{35}$ Nevertheless, the increase in psychiatric outpatient visits shows that the general population needed psychiatric help during the pandemic. In a study of US adults with no history of mental illness, more than one in four people suffered psychological distress in the early stages of the COVID-19 pandemic. ${ }^{36}$ In a study conducted in Spain on the general population, approximately $1 / 3$ (one third) of the participants reported substantial psychological impact, specifically showing 
high rates of depression and stress. ${ }^{37}$ Our study shows that the burden of mental health during the COVID-19 pandemic has increased in Korea, and people have made a psychiatric visit for treatment.

Our study focused on patients who were tested for SARSCoV-2. During the COVID-19 pandemic, the general population has suffered from a loss of daily life and reduced access to favored physical activities. ${ }^{19}$ In addition, those who were tested for SARS-CoV-2 were quarantined at home or at a quarantine facility because of the possibility of having contact with confirmed positive COVID-19 cases in Korea. These individuals may be more vulnerable to worsened mental illness because quarantine is associated with deterioration of mental health (e.g., depression, anxiety, insomnia, lack of self-control, and lower self-esteem). ${ }^{38}$

Regarding the mental health of those with pre-existing mental illnesses, most studies reported that patients with pre-existing mental illness experienced more psychological symptoms and worsened mental health during COVID-19. ${ }^{16,39}$ Unexpectedly, our findings showed that patients with pre-existing mental illness significantly decreased in psychiatric outpatients during the COVID-19 pandemic. Seo and colleagues ${ }^{40}$ similarly found that the number of outpatient visits decreased in a tertiary hospital. The daily visit rates for anxiety, depression, and schizophrenia spectrum were significantly reduced by approximately $29.8 \%, 14.8 \%$, and $13.3 \%$, respectively. In addition, a Japanese study found a decrease in the number of outpatients in psychiatric hospitals. ${ }^{41}$ These results suggest that patients with pre-existing mental illness tend to be more reluctant to visit hospitals. Some people with high anxiety may be hesitant to seek medical treatment since they consider hospitals to be places where they could be contacted with a contagious virus. ${ }^{42}$ Moreover, social distancing might have changed daily lives and reduced social support in psychiatric patients during the COVID-19 pandemic. Consequently, Kozloff et al. ${ }^{43}$ suggest that those with a mental disorder are at high risk of worsening symptoms and can lead to social isolation, which can eventually lead to missed treatment. In addition, the decrease in psychiatric outpatient visits during the COVID-19 pandemic was more pronounced in the COVID-19 positive patients among those with pre-existing mental illness, suggesting that they were physically unable to visit psychiatric outpatient clinics because of COVID-19 treatment.

We found that among those who were tested for SARS$\mathrm{CoV}-2$, the number of psychiatric inpatients noticeably increased in April 2020 during the initial phase of COVID-19 pandemic. Moreover, among these individuals, the number of outpatient visits peaked in March, while inpatient visits peaked in April. This shows that the spread of the virus may increase the risk of recurrence due to enhanced social dis- tancing and the lack of adequate medical services due to the closure of some medical centers. A study from Japan showed that the length of hospitalization for mental health care increased during COVID-19. ${ }^{41}$ In addition, another study found that psychiatric inpatients increased by $21.4 \%$ among those over 65 years of age following the COVID-19 outbreak. This increase was greater and lasted longer than SARS, and the study reported that it was important to maintain support to reduce the risk of worsening mental health during COVID-19.

According to our results, the number of inpatient visits has increased significantly compared to the same month of the previous year. Among the "negative for SARS-CoV-2" group, the number of inpatient visits increased until April and decreased in May. Whereas, in the case of the "positive for SARSCoV-2" group, the number of inpatient visits continued to increase until May. This means that more psychiatric care was needed for the COVID-19 positive patients. Previous studies have shown that infections are associated with a higher risk of mood disorders, ${ }^{45}$ and there have been reports of more frequent depressive symptoms in infected people during SARS$\mathrm{CoV}-1$ epidemic. ${ }^{10}$ In addition, a study shows that the level of posttraumatic stress symptoms is significantly higher among patients hospitalized with COVID-19.7 This may have been due to reasons such as stigma, social isolation, uncertainty in treatment, fear of viral transmission, and negative news which leads positive patients to experience loneliness, depression, anxiety, anger, insomnia and posttraumatic stress symptoms. ${ }^{46}$ Another explanation is that there were many confirmed COVID-19 positive cases at psychiatric hospitals (i.e., Cheongdo Daenam Hospital $)^{27}$ in the early days of COVID-19. For this reason, among our data, the "positive for SARS-CoV-2" group included a lot of serious patients who had been hospitalized in psychiatric hospitals, and it is presumed that they required subsequent psychiatric hospitalization after treatment for COVID-19.

Finally, we analyzed the number of psychiatric medication prescriptions from January 2019 to May 2020. Our results indicate that all psychiatric medication prescriptions were peaked between March and April 2020. Sedative-hypnotic prescriptions, in particular, were prescribed most frequently in March. These patterns tended to be similar to those of psychiatric outpatient visits. It is notable that among the "positive for SARSCoV-2" group, the number of psychiatric medication prescriptions continued to increase until May. These patterns tended to be similar to those of psychiatric inpatient visits among the COVID-19 positive patients. A study in the United States showed that the number of prescriptions for antidepressants, antianxiety, and anti-insomnia increased by $21 \%$ during the COVID-19 pandemic between February and March 2020. ${ }^{47}$ Likewise, studies conducted in England confirmed that the 
highest number of antidepressants was observed between February and March $2020 .^{48}$ In addition, Keller et al. ${ }^{49}$ found an increasing sedative-hypnotic prescription rate and a significant increase in prescriptions after the second state shutdown. Because of sustained stress, patients may have demanded more sedative hypnotics.

Our results indicate an increased need for psychiatric medication during the first COVID-19 outbreak in Korea, especially in COVID-19 positive patients. Many factors may have led to an increase in psychiatric prescriptions, most of which may be due to the increase and deterioration of mental health problems (e.g., anxiety, depression, stress, sleep disorder, etc.) during the COVID-19 pandemic. ${ }^{47,50}$ Medications are prescribed to relieve psychological symptoms during the COVID-19 pandemic; however, side effects can occur if the medication is taken over a long period of time. Therefore, care should be taken to avoid overdose. ${ }^{51}$

Despite the meaningful findings, this study has some limitations. First, since this study was conducted on all people who tested for SARS-CoV-2 for a certain period of time, the results are representative of Korean people who tested for COVID-19, but cannot be generalized to the general population. Second, the data included individuals who tested for SARS-CoV-2 from January 2020 to May 2020. The results are related only to the early phases of the pandemic. Therefore, it is necessary to replicate these findings in later periods through additional data. Third, in the case of medications, though other studies ${ }^{27,52}$ have analyzed them based on the amount of prescribed medications, we only examined the number of psychiatric medication prescription record for each four medication categories.

In conclusion, we identified that the number of psychiatric visits increased during the initial phase of COVID-19 pandemic. This pattern was particularly noticeable in patients who did not have pre-existing mental illness before the COVID-19 pandemic, which means that mental problems were newly developed during the COVID-19 outbreak. For patients with pre-existing mental illness, especially those who tested positive for SARS-CoV-2, the decrease in outpatient visits, worsening symptoms, and a subsequent increase in hospitalization seem to be noteworthy problems. In addition, we identified that the prescriptions of psychiatric medications were higher as compared to COVID-19 among those who were tested for SARS-CoV-2. However, our study was only able to confirm the trends in psychiatric visits and medication prescription patterns during the first wave of the COVID-19 pandemic, and further research is needed to evaluate how these results change. With the prolonged COVID-19 pandemic, mental problems are expected to worsen or increase in the future. Therefore, government efforts are needed to reduce the mental health issues prevalent in our society and maintain mental

health service use among patients with pre-existing severe mental illness during the COVID-19 pandemic.

\section{Availability of Data and Material}

The datasets generated or analyzed during the current study are available in the National Health Insurance Service (NHIS) repository, https:// nhiss.nhis.or.kr/bd/ay/bdaya001iv.do.

\section{Conflicts of Interest}

Subin Park, a contributing editor of the Psychiatry Investigation, was not involved in the editorial evaluation or decision to publish this article. All remaining authors have declared no conflicts of interest.

\section{Author Contributions}

Conceptualization: Subin Park. Data curation: Minkyung Jo. Formal analysis: Min Geu Lee. Investigation: Jungeun Kim, Soo Jung Rim. Methodology: Jungeun Kim. Project administration: Subin Park. Supervision: Subin Park, Minkyung Jo. Visualization: Jungeun Kim. Writing-original draft: Jungeun Kim, Soo Jung Rim, Subin Park. Writing_review \& editing: Jungeun Kim, Soo Jung Rim, Subin Park.

\section{ORCID iDs}

Jungeun Kim

Soo Jung Rim

Minkyung Jo

Min Geu Lee

Subin Park

\begin{abstract}
https://orcid.org/0000-0002-5457-745X https://orcid.org/0000-0003-4938-4116 https://orcid.org/0000-0002-4208-0024 https://orcid.org/0000-0003-2916-7421 https://orcid.org/0000-0002-4623-9899
\end{abstract}

\section{Funding Statement}

This study was supported by an Intramural Research Grant (No. R201906) from the National Center for Mental Health, Ministry of Health \& Welfare, Korea.

\section{REFERENCES}

1. WHO Coronavirus Disease (COVID-19) Dashboard. Available at: https://covid19.who.int/. Accessed July 16, 2021.

2. KCDC. The updates on COVID-19 in Korea as of Feb 25. Seoul: Korea Centers for Disease Control and Prevention; 2020.

3. Kim YJ, Sung H, Ki CS, Hur M. COVID-19 Testing in South Korea: current status and the need for faster diagnostics. Ann Lab Med 2020; 40:349-350.

4. Coronavirus disease-19, Republic of Korea, 2021. Available at: http:// ncov.mohw.go.kr/en/baroView.do?brdId=11\&brdGubun=111\&dataG ubun=\&ncrContSeq=\&contSeq=\&board_id=. Accessed July 16, 2021.

5. Lee HS, Dean D, Baxter T, Griffith T, Park S. Deterioration of mental health despite successful control of the COVID-19 pandemic in South Korea. Psychiatry Res 2021;295:113570.

6. Pfefferbaum B, North CS. Mental health and the COVID-19 pandemic. N Engl J Med 2020;383:510-512.

7. Bo HX, Li W, Yang Y, Wang Y, Zhang Q, Cheung T, et al. Posttraumatic stress symptoms and attitude toward crisis mental health services among clinically stable patients with COVID-19 in China. Psychol Med 2021;51:1052-1053.

8. Rossi R, Socci V, Talevi D, Mensi S, Niolu C, Pacitti F, et al. COVID-19 pandemic and lockdown measures impact on mental health among the general population in Italy. Front Psychiatry 2020;11:790.

9. Cheng SK, Tsang JS, Ku KH, Wong CW, Ng YK. Psychiatric complications in patients with severe acute respiratory syndrome (SARS) during the acute treatment phase: a series of 10 cases. Br J Psychiatry 2004; 184:359-360.

10. Chua SE, Cheung V, McAlonan GM, Cheung C, Wong JW, Cheung EP, et al. Stress and psychological impact on SARS patients during the out- 
break. Can J Psychiatry 2004;49:385-390.

11. Jalloh MF, Li W, Bunnell RE, Ethier KA, O’Leary A, Hageman KM, et al. Impact of Ebola experiences and risk perceptions on mental health in Sierra Leone, July 2015. BMJ Glob Health 2018;3:e000471.

12. Hawryluck L, Gold WL, Robinson S, Pogorski S, Galea S, Styra R. SARS control and psychological effects of quarantine, Toronto, Canada. Emerg Infect Dis 2004;10:1206-1212.

13. Mak IW, Chu CM, Pan PC, Yiu MG, Chan VL. Long-term psychiatric morbidities among SARS survivors. Gen Hosp Psychiatry 2009;31:318326.

14. Lam MH, Wing YK, Yu MW, Leung CM, Ma RC, Kong AP, et al. Mental morbidities and chronic fatigue in severe acute respiratory syndrome survivors: long-term follow-up. Arch Intern Med 2009;169:21422147.

15. Vindegaard N, Benros ME. COVID-19 pandemic and mental health consequences: systematic review of the current evidence. Brain Behav Immun 2020;89:531-542.

16. Zhou J, Liu L, Xue P, Yang X, Tang X. Mental health response to the COVID-19 outbreak in China. Am J Psychiatry 2020;177:574-575.

17. Brooks SK, Webster RK, Smith LE, Woodland L, Wessely S, Greenberg $\mathrm{N}$, et al. The psychological impact of quarantine and how to reduce it: rapid review of the evidence. Lancet 2020;395:912-920.

18. Zhang J, Lu H, Zeng H, Zhang S, Du Q, Jiang T, et al. The differential psychological distress of populations affected by the COVID-19 pandemic. Brain Behav Immun 2020;87:49-50.

19. Dean DJ, Tso IF, Giersch A, Lee HS, Baxter T, Griffith T, et al. Crosscultural comparisons of psychosocial distress in the USA, South Korea, France, and Hong Kong during the initial phase of COVID-19. Psychiatry Res 2021;295:113593.

20. Jacob L, Smith L, Armstrong NC, Yakkundi A, Barnett Y, Butler L, et al. Alcohol use and mental health during COVID-19 lockdown: a crosssectional study in a sample of UK adults. Drug Alcohol Depend 2021; 219:108488.

21. Kang E, Lee SY, Kim MS, Jung H, Kim KH, Kim KN, et al. The psychological burden of COVID-19 stigma: evaluation of the mental health of isolated mild condition COVID-19 patients. J Korean Med Sci 2021;36: e33.

22. Pierce M, Hope H, Ford T, Hatch S, Hotopf M, John A, et al. Mental health before and during the COVID-19 pandemic: a longitudinal probability sample survey of the UK population. Lancet Psychiatry 2020;7:883-892.

23. Shi L, Lu ZA, Que JY, Huang XL, Liu L, Ran MS, et al. Prevalence of and risk factors associated with mental health symptoms among the general population in China during the coronavirus disease 2019 pandemic. JAMA Netw Open 2020;3:e2014053.

24. Castro VM, Perlis RH. Electronic health record documentation of psychiatric assessments in Massachusetts emergency department and outpatient settings during the coronavirus disease 2019 (COVID-19) pandemic. JAMA Netw Open 2020;3:e2011346.

25. Cheol Seong S, Kim YY, Khang YH, Heon Park J, Kang HJ, Lee H, et al. Data resource profile: The National Health Information Database of the National Health Insurance Service in South Korea. Int J Epidemiol 2017; 46:799-800.

26. WHO Diagnostic testing for SARS-CoV-2. Available at: https://apps. who.int/iris/rest/bitstreams/1302661/retrieve. Accessed July 16, 2021.

27. Jung SJ, Lee J, Choi JW, Kim S, Shin A, Lee YJ. Association between sedative-hypnotic medication use and incidence of cancer in Korean Nation Health Insurance Service data. Sleep Med 2019;60:159-164.

28. Butt AA, Azad AM, Kartha AB, Masoodi NA, Bertollini R, Abou-Samra $A B$. Volume and acuity of emergency department visits prior to and after COVID-19. J Emerg Med 2020;59:730-734.

29. Hyun J, You S, Sohn S, Kim SJ, Bae J, Baik M, et al. Psychosocial support during the COVID-19 outbreak in Korea: activities of multidisciplinary mental health professionals. J Korean Med Sci 2020;35:e211.

30. Kim T. Improving preparedness for and response to coronavirus dis- ease 19 (COVID-19) in long-term care hospitals in Korea. Infect Chemother 2020;52:133-141.

31. Park SY, Kim YM, Yi S, Lee S, Na BJ, Kim CB, et al. Coronavirus disease outbreak in call center, South Korea. Emerg Infect Dis 2020;26:16661670.

32. Han T. Outbreak investigation: transmission of COVID-19 started from a spa facility in a local community in Korea. Epidemiol Health 2020;42:e2020056.

33. Choi JY. COVID-19 in South Korea. Postgrad Med J 2020;96:399-402.

34. Ettman CK, Abdalla SM, Cohen GH, Sampson L, Vivier PM, Galea S. Prevalence of depression symptoms in US adults before and during the COVID-19 pandemic. JAMA Netw Open 2020;3:e2019686.

35. Roh S, Lee SU, Soh M, Ryu V, Kim H, Jang JW, et al. Mental health services and R\&D in South Korea. Int J Ment Health Syst 2016;10:45.

36. Holingue C, Badillo-Goicoechea E, Riehm KE, Veldhuis CB, Thrul J, Johnson RM, et al. Mental distress during the COVID-19 pandemic among US adults without a pre-existing mental health condition: Findings from American trend panel survey. Prev Med 2020;139:106231.

37. Rodríguez-Rey R, Garrido-Hernansaiz H, Collado S. Psychological impact and associated factors during the initial stage of the coronavirus (COVID-19) pandemic among the general population in Spain. Front Psychol 2020;11:1540.

38. Hossain MM, Sultana A, Purohit N. Mental health outcomes of quarantine and isolation for infection prevention: a systematic umbrella review of the global evidence. Epidemiol Health 2020;42:e2020038.

39. Chatterjee SS, Barikar CM, Mukherjee A. Impact of COVID-19 pandemic on pre-existing mental health problems. Asian J Psychiatr 2020; 51:102071.

40. Seo JH, Kim SJ, Lee M, Kang JI. Impact of the COVID-19 pandemic on mental health service use among psychiatric outpatients in a tertiary hospital. J Affect Disord 2021;290:279-283.

41. Makiyama K, Kawashima T, Nomura S, Eguchi A, Yoneoka D, Tanoue $\mathrm{Y}$, et al. Trends in healthcare access in Japan during the first wave of the COVID-19 pandemic, up to June 2020. Int J Environ Res Public Health 2021;18:3271.

42. Asmundson GJG, Taylor S. How health anxiety influences responses to viral outbreaks like COVID-19: What all decision-makers, health authorities, and health care professionals need to know. J Anxiety Disord 2020;71:102211.

43. Kozloff N, Mulsant BH, Stergiopoulos V, Voineskos AN. The COVID-19 global pandemic: implications for people with schizophrenia and related disorders. Schizophr Bull 2020;46:752-757.

44. Lee ATC, Mo FYM, Lam LCW. Higher psychogeriatric admissions in COVID-19 than in severe acute respiratory syndrome. Int J Geriatr Psychiatry 2020;35:1449-1457.

45. Benros ME, Waltoft BL, Nordentoft M, Ostergaard SD, Eaton WW, Krogh J, et al. Autoimmune diseases and severe infections as risk factors for mood disorders: A Nationwide Study. JAMA Psychiatry 2013; 70:812-820.

46. Xiang YT, Yang Y, Li W, Zhang L, Zhang Q, Cheung T, et al. Timely mental health care for the 2019 novel coronavirus outbreak is urgently needed. Lancet Psychiatry 2020;7:228-229.

47. America's state of mind report. Available at: https://www.expressscripts.com/corporate/americas-state-of-mind-report? $r=w w w \_e x-$ pressscripts_com. Accessed July 2, 2021.

48. Rabeea SA, Merchant HA, Khan MU, Kow CS, Hasan SS. Surging trends in prescriptions and costs of antidepressants in England amid COVID-19. DARU J Pharm Sci 2021;29:217-221.

49. Keller MS, Kiefer E, Campbell S, Bradley K, Mashburn R, Bawa M, Goldzweig C. Sustained increase of sedative-hypnotic prescribing during the COVID-19 pandemic in a large urban health system: an observational study. J Gen Intern Med 2021;36:3618-3620.

50. Gautam S, Jain A, Gautam M, Vahia VN, Gautam A. Clinical practice guidelines for the management of generalised anxiety disorder (GAD) and panic disorder (PD). Indian J Psychiatry 2017;59(Suppl 1):S67-S73. 
51. Brandt J, Leong C. Benzodiazepines and Z-drugs: an updated review of major adverse outcomes reported on in epidemiologic research. Drugs R D 2017;17:493-507.
52. Tachkov K, Ignatova D, Mitov K, Kamusheva M, Petrova G. Trends in the cost and utilization of psychotropic medicines for major psychiatric disorders in Bulgaria from 2013 to 2017. Eur J Psychiatry 2021;35:33-40. 